\title{
AR Display Method of a Person's Identifier near the Head on a Camera Screen Based on the GPS Information and Face Detection Using Ad hoc and P2P Networking
}

\author{
Masahiro Gotou and Kazumasa Takami \\ Department of Information Systems Science, Faculty of Engineering, SOKA University, Hachioji-shi, Tokyo 192-8577, Japan
}

\begin{abstract}
The spread of social media has increased contacts of members of communities on the Internet. Members of these communities often use account names instead of real names. When they meet in the real world, they will find it useful to have a tool that enables them to associate the faces in front of them with the account names they know. This paper proposes a method that enables a person to identify the account name of the person ("target") in front of him/her using a smartphone. The attendees to a meeting exchange their identifiers (i.e., the account name) and GPS information using smartphones. When the user points his/her smartphone towards a target, the target's identifier is displayed near the target's head on the camera screen using AR (augmented reality). The position where the identifier is displayed is calculated from the differences in longitude and latitude between the user and the target and the azimuth direction of the target from the user. The target is identified based on this information, the face detection coordinates, and the distance between the two. The proposed method has been implemented using Android terminals, and identification accuracy has been examined through experiments.
\end{abstract}

Key words: Ad hoc networking, AR (augmented reality), face detection, GPS information, person's identifier, P2P communication, smartphone.

\section{Introduction}

The penetration of the Internet has given rise to a growth in social media, such as Twitter, Facebook, and LINE. The percentage of social media usage now exceeds $60 \%$ [1]. Community members increasingly get together in the real world. According to a survey conducted in fiscal 2010 [2], 45.9\% of the respondents who belong to an SNS, and $62.2 \%$ of those who access an SNS daily had attended offline meetings. Such meetings have become part of their lives. However, since most people in an offline meeting meet for the first time, they find it difficult to identify other attendees before they introduce themselves. One solution proposed was NameTag [3], which requires each member to attach a face photo to his/her icon or

Corresponding author: Kazumasa Takami, doctor of engineering, professor, research fields: self-organized network, intelligent software system, ad hoc communications, P2P services. profile in social media and uses these photos to identify attendees to an offline meeting using facial recognition technology. However, this method cannot be useful if many members hesitate to disclose their face photos to the public. Another idea is to require members to wear nametags that contain their business cards but this cannot be useful if the fonts on business cards are too small.

Since smartphones have become widespread and its computing power has risen, applications have been developed for personal identification using sensors, GPS and AR (augmented reality) [4-6]. Several services, such as "Sekai camera [7]," "Layar [8]," display information that is derived from GPS data, and tags either printed on books or attached on objects using AR. However, these services can display only large buildings, such as stores, commercial buildings or other landmarks, which can be easily identified from GPS information, or objects to which tags were 
attached in advance.

There is a study on an opportunistic network [9-14], which is an information communication network that uses terminals owned by people who pass by in daily lives as nodes. A software program that has a passer-by communication function is provided for Android terminals. It allows the user to send tweets, etc. to get in touch with the persons who passed by $\mathrm{him} / \mathrm{her}$ on the street. However, if the user is to exchange, with confidence, information with a person whose face photo is not disclosed, some means should be available to identify the passers-by.

This paper proposes a method that enables the user to know the identity of people in close proximity using a smartphone. The smartphones of the people concerned exchange messages that contain the identifier (i.e., account name in a community on the Internet) and GPS location information. The identifier is displayed near the head of the target person on the camera screen using AR. This will enable users to easily identify those people whom they know by their account names on the Internet but have not met physically. This method involves two procedures. One is calculation of the identifier display coordinates from differences in longitude and latitude between the locations of the user and the target person and the azimuth direction of the target from the user. This was already proposed by the authors in Ref. [15]. This is supplemented by the second procedure, which uses the distance between the coordinates obtained above and the face detection coordinates. We implemented this method in Android smartphones and examined the accuracy of identifying target persons through experiments. Section 2 presents a conceptual model of the proposed method, and describes how the GPS coordinates and face detection coordinates of the target person is used to display the identifier of the target person. Section 3 describes the configuration of the experimental system we developed using Android terminals. Section 4 discusses experiment results. Section 5 gives conclusions and future issues.

\section{Displaying the Identifier of the Target Person near His/Her Head Based on His/Her GPS Coordinates and Face Detection Coordinates}

This section describes how the identifier of the target person can be displayed near his/her head on a camera screen using AR. A conceptual model is used to explain the functions of this method. Three technical issues are discussed. The first is how to improve the accuracy of a person's location information derived from GPS coordinates. The second is how to calculate the relative spatial positions from differences in longitude and latitude between the user and the target and the azimuth direction of the target from the user. The third is how to correct the identifier display position from the face detection coordinates.

\subsection{Conceptual Model of the Proposed Method}

A model for displaying a person's identifier on the camera screen is presented here. Three types of coordinates are considered: the GPS coordinates of a person, the coordinates of the position at which a person's face is detected through a camera (face detection coordinates), and the position at which the person's identifier should be displayed (identifier display coordinates).

Three patterns are considered with regard to the number of targets and the number of persons involved. Let the identifier of a target persons be $s_{-} i d$, the GPS coordinates of $\mathrm{s}$ be $s_{-} G P S_{-}$coordinate, and the identifier display coordinates of $s$ be $P_{s}$. Let the face detection coordinates of an unknown person be $F_{u k}$. After the person is identified as $s, F_{u k}$ changes to $F_{s}$. The persons involved exchange messages using $\mathrm{P} 2 \mathrm{P}$ communication. Each message contains the $s_{-} i d$ and s_GPS_coordinate of the owner $s$ of the sending terminal.

2.1.1 Pattern 1: Only One Target Person

Only Target B is near User A. A's camera screen displays the face of B. This pattern is shown in Fig. 1 . 
B's terminal sends a message to A. Since both $F_{u k}$ and $P_{B}$ exist within A's camera screen, it is determined that $F_{u k}$ is B, and identifier ( $B \_i d$ ) is displayed near the head of $F_{B}$.

2.1.2 Pattern 2: Only One Target Person but the Faces of Two Persons Are Detected

Target $\mathrm{B}$ and non-target $\mathrm{C}$ are near User $\mathrm{A}$. B sends a message to A. A's terminal detects two faces. This pattern is shown in Fig. 2.

$F_{u k 1}, F_{u k 2}$ and $P_{B}$ are within A's camera screen. The distance between $P_{B}$ and $F_{u k l}$ and the distance between $P_{B}$ and $F_{u k 2}$ are compared. Since $F_{u k l}$ is closer to $P_{B}$ than $F_{u k 2}$ is, it is determined that $F_{u k l}$ is $\mathrm{B}$, and identifier $\left(B \_i d\right)$ is displayed near the head of $F_{B}$.

2.1.3 Pattern 3: Two Target Persons and the Faces of Two Persons are detected

Both Targets B and C are near User A. Both of them send a message to A. A's terminal detects two faces. This pattern is shown in Fig. 3. $F_{u k l}, F_{u k 2}, P_{B}$ and $P_{C}$ are within A's camera screen. The distance between $P_{B}$ and $F_{u k l}$ and the distance between $P_{B}$ and $F_{u k 2}$ are compared. $P_{B}$ is closer to $F_{u k l}$ than to $F_{u k 2}$. Therefore, it is determined that $F_{u k l}$ is B. Likewise, distance between $P_{C}$ and $F_{u k l}$ and the distance between $P_{C}$ and $F_{u k 2}$ are compared. Since $P_{C}$ is closer to $F_{u k 2}$ than to $F_{u k l}$, it is determined that $F_{u k 2}$ is C. Thus, identifiers of $B \_i d$ and $C \_i d$ are displayed near the heads of the respective persons.

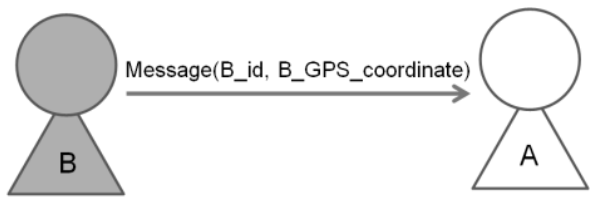

(a) Send a P2P message

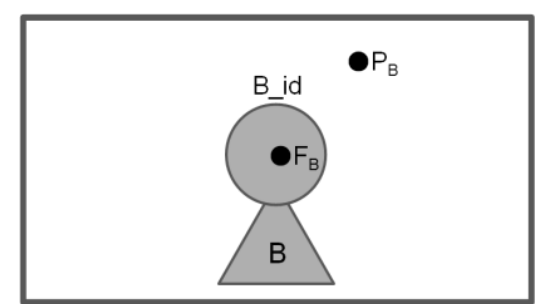

(b) A's camera screen

(note) s_id: identifier of s, S_GPS_coordinate: location information of $\mathrm{s}$, $P_{\mathrm{s}}$ : identifier display coordinates calculated from $s=G P S \_$coordinate, $F_{s}$ : face detection coordinates of $s$

Fig. 1 Only one target person.

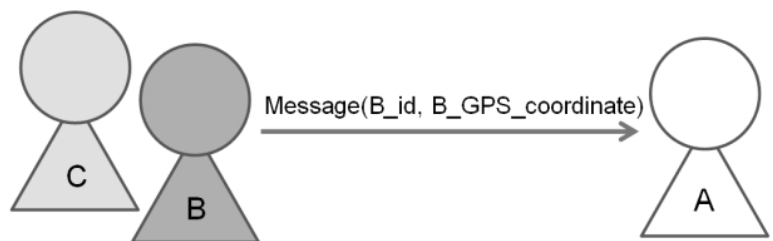

(a) Send a P2P message

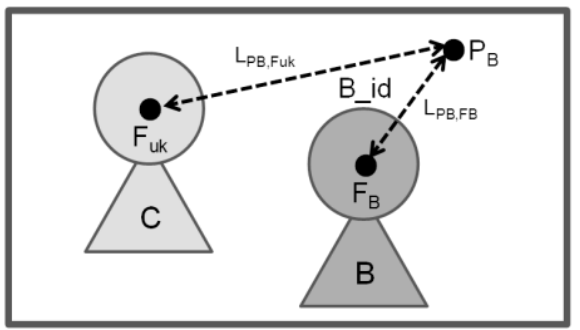

(b) A's camera screen $\left(L_{P B, F B}<L_{P B, F u k}\right)$

(note) ) s_id: identifier of s, S_GPS_coordinate: location information of $\mathrm{s}$, $P_{s}$ : identifier display coordinates calculated from $s=$ GPS_coordinate, $F_{s}$ : face detection coordinates of $s, L_{P_{s F s}}$ : distance between $P_{s}$ and $F_{s}$

Fig. 2 Only one target person but the faces of two persons are detected.

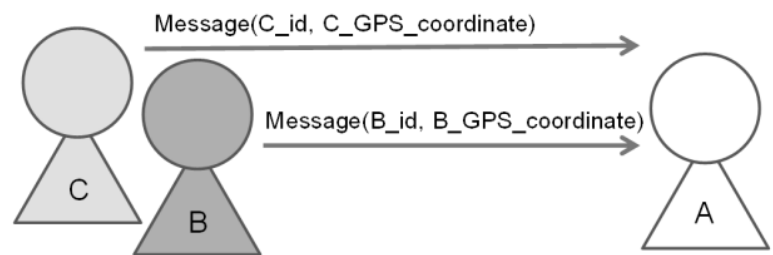

(a) Send a P2P message

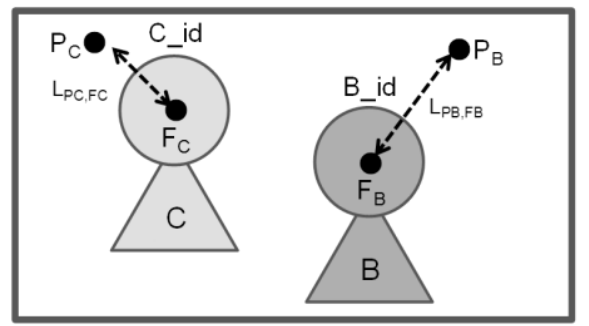

(b) A's camera screen

(note) ) s_id: identifier of S, S_GPS_coordinate: location information of s, $P_{s}$ : identifier display coordinates calculated from $s=$ GPS coordinate, $F_{s}$ : face detection coordinates of $s, L_{P_{s} F_{s}}$ : distance between $P_{s}$ and $F_{s}$

Fig. 3 Two target persons and the faces of two persons are detected.

\subsection{Use of a Center-Of-Gravity Model to Compensate for GPS Measurement Errors}

To identify the target persons on the camera screen correctly, it is important to ensure that GPS measurements are accurate. To compensate for GPS measurement errors, we use a center-of-gravity model, which the authors proposed in Ref. [15]. This model assumes that the user and the targets are close enough 
to see each other, and that they share the same GPS measurement conditions, such as the weather and other environmental conditions and the number of accessible satellites.

Consider the center of gravity of a triangle made up of three GPS coordinates of the same person measured within a relatively short span. We regard that the person is located at the center of gravity. As shown in Fig. 4, an advantage of this model is that the error of the center of gravity is always equal to or smaller than the maximum error of any point even though it may be greater than the error of one or two points.

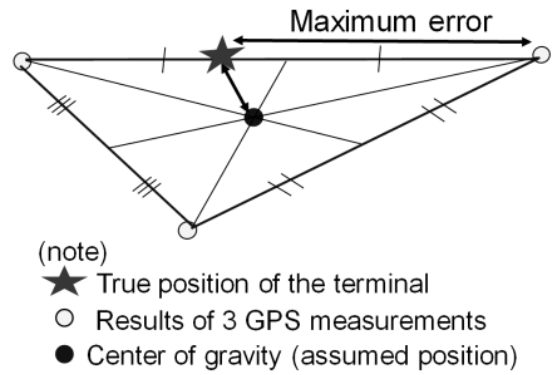

Fig. 4 Calculation of a target person's location using the center-of-gravity model.

\subsection{Calculation of the Identifier Display Coordinates} from Differences in Longitude and Latitude between the Two Persons and the Azimuth Direction (Relative Spatial Position)

The terminals of the persons involved exchange their GPS location information obtained in the manner described in Section 2.2. We calculate the relative spatial position of the two terminals, and calculate identifier display coordinates $P_{s}(x, y)$, the position at which identifier $s_{-} i d$ is displayed. The algorithm that was proposed by the authors in Ref. [15] is used for this calculation. This algorithm is described below. Refer to the relative spatial position plane shown in Fig. 5.

Step 1: Calculate the differences in longitude and latitude between the GPS locations of the user and the target. Create a relative spatial position plane. Its vertical axis shows the difference in latitude, and the horizontal axis shows the difference in longitude. The user is at the origin. Let the coordinates of the target $s$ be $R_{s}(x, y)$. Calculate $R_{s}(x, y)$ and determine the quadrant in which the target is.

Step 2: Consider a point $(H)$ on the horizontal axis at which it intersects with a line that goes through $\mathrm{s}$ and is parallel to the vertical axis. Create a triangle with vertices $\mathrm{U}, \mathrm{S}$ and $\mathrm{H}$. Calculate the angle $\delta(=\arctan (y / x))$ between the oblique line of this triangle and the horizontal axis.

Step 3: Calculate angle $\alpha$ between the direction of north (which is the reference direction) and the target based on the quadrant in which angle $\delta$ and the target exist.

3-1: If the target is in the second or third quadrant, $\alpha=90^{\circ}+(-1)^{n-1} \times \delta$ ( $n$ is the quadrant number);

3-2: If the target is in the first or fourth quadrant, $\alpha=270^{\circ}+(-1)^{n-1} \times \delta$ ( $\mathrm{n}$ is the quadrant number).

Step 4: Using an electronic compass, measure angle $\beta$ between the direction of north and the direction in which the user is facing. Calculate the difference in angle between the direction in which the user is facing and the direction of the target: $\Delta \mathrm{us}(=\beta-\alpha)$.

Step 5: Calculate $P_{s}\left(x, y_{f i x}\right)$ from the fact that the direction of the target differs from the direction in which the user is facing by angle $\Delta$ us. The $\mathrm{x}$ coordinate of $P_{s}\left(x, y_{f i x}\right)$ is calculated using Eq. (1). The y coordinate is fixed at $y_{f i x}$ to avoid the influences of shaking of the camera.

$$
\mathbf{x}=\Delta \mathrm{us} \times \mathrm{K}
$$

where $\mathrm{K}$ is a constant that depends on the camera screen size and the viewing angle.

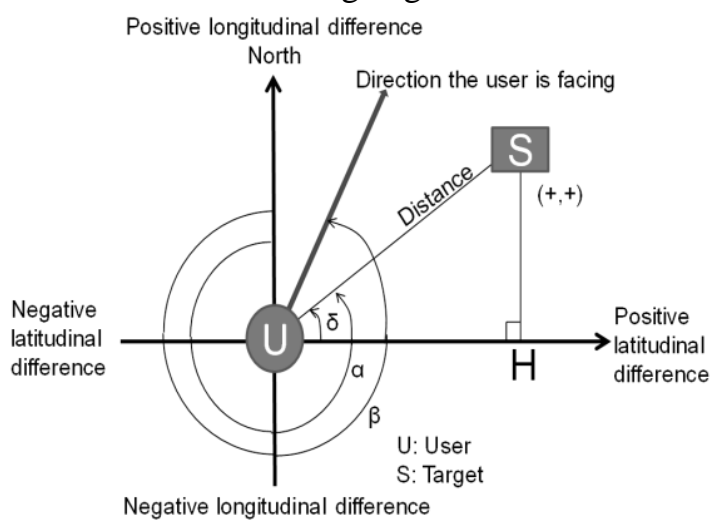

Fig. 5 Relative spatial position plane. 


\subsection{Correction of the Identifier Display Position} Based on Face Detection Coordinates

This section describes the algorithm for determining the $s \_i d$ display position from $P_{s}(x, y)$ calculated in Section 2.3 and $F_{u k}(x, y)$ of the unknown person.

Step 1: The user receives $m$ messages and detects $k$ faces.

Step 2: Calculate $m P_{s}(x, y)$, and calculate its set $\left\{\mathrm{P}_{\mathrm{si}}\left(\mathrm{x}_{\mathrm{i}}, \mathrm{y}_{\mathrm{i}}\right) \mid \mathrm{i}=1 \sim m\right\}$.

Step 3: Calculate set $\left\{\mathrm{F}_{\mathrm{ukj}}\left(\mathrm{x}_{\mathrm{j}}, \mathrm{y}_{\mathrm{j}}\right) \mid j=1 \sim k\right\}$ of $k$ face detection coordinates.

Step 4: Calculate the distances of all combinations of $P_{s i}\left(x_{i}, y_{i}\right)$ and $F_{u k j}\left(x_{j}, y_{j}\right)$. Let the set of these distances be $\left\{\mathrm{L}_{\mathrm{si}, \text { ukj }} \mid \mathrm{i}=1 \sim m, \mathrm{j}=1 \sim k\right\}$.

Step 5: Determine the correspondence between the targets and the faces detected. The candidate of Target $s_{i}$ is stored in $C_{s i}\left(F_{u k j}, L_{s i, u k j}\right)$.

5-1: Set $\mathrm{j}=1$, search column set $\left\{\mathrm{L}_{\mathrm{si}, \mathrm{uk} 1} \mid \mathrm{i}=1 \sim m, \mathrm{j}=1\right\}$, and find $s_{w}$ that has the minimum value. This $s_{w}$ is considered the candidate, and is set in $C_{s w}\left(F_{u k l}, L_{s w, u k l}\right)$.

5-2: Set $\mathrm{j}=2$ and perform the following for $s_{w}$, which already has a candidate value.

Case 1: If $L_{s w, u k 2} \geq C_{s w}\left(L_{s w, u k 1}\right)$ then retain $C_{s w}$.

Search column set $\left\{\mathrm{L}_{\mathrm{si}, \mathrm{uk} 2} \mid \mathrm{i}=1 \sim m, \quad \mathrm{i} \neq w, \quad \mathrm{j}=2\right\}$ excluding $s_{w}$, and find $s_{z}$ that has the minimum value. This $s_{z}$ is considered the candidate, and is set in $C_{s z}\left(F_{u k 2}, L_{s z, u k 2}\right)$.

Case 2: If $L_{s w, u k 2}<C_{s w}\left(L_{s w, u k l}\right)$ then discard $C_{s w}$.

Search column set $\left\{\mathrm{L}_{\mathrm{si}, \mathrm{uk} 2} \mid \mathrm{i}=1 \sim m, \mathrm{j}=2\right\}$, and find $s_{z}$ that has the minimum value. This $s_{z}$ is considered the candidate, and is set in $C_{s z}\left(F_{u k 2}, L_{s z, u k 2}\right)$.

5-3: Repeat 5-2 for $\mathrm{j}=3$ to $k$.

5-4: Check whether candidates are found for all $s_{i}$.

Case 1: If yes, go to Step 6.

Case 2: No.

Case2-1: There is a competitor (target $s_{i}$ ). Go to Step 5-1.

Case2-2: There is no competitor, and only $s_{v}$ remains. $F_{u k q}$, which has not been associated with any target, is selected as the candidate for $s_{v}$ and is set in
$C_{s v}\left(F_{u k q}, L_{s v, u k q}\right)$. Go to Step 6.

Step 6: The identifier $s_{i-} i d$ is to be displayed near the head of $F_{u k j}$ of $C_{s i}\left(F_{u k j}\right)$. This is repeated for $\mathrm{i}=1$ to $m$.

Two examples of the above algorithm are described below for cases where $m=2$ and $k=2$. The distance between the coordinates is different between the two examples.

(Example 1)

Steps 1 to 4: Generate set $\left\{L_{s i, u k j} \mid i=1 \sim 2, j=1 \sim 2\right\}$. The value of each element is shown below.

\begin{tabular}{|c|c|c|}
\hline $\begin{array}{ll}\text { Target } s & \text { Face detected } u k \\
\end{array}$ & $u k l$ & $u k 2$ \\
\hline$s 1$ & 2 & 4 \\
\hline$s 2$ & 3 & 1 \\
\hline
\end{tabular}

Step 5-1: Search column $u k 1$ for the minimum value. Target $s 1$ has the minimum value. Generate $\mathrm{C}_{\mathrm{s} 1}=(u k 1,2)$. Nothing is done on $s 2$.

Step 5-2 Case 1: Move to column $u k 2$. Since $s 1$ has a candidate value, compare $L_{s 1, u k 2}=4$ with $C_{s I}($ distance value $)=2$. Since $C_{s I}($ distance value $)$ is smaller, candidate value $C_{s l}=(u k l, 2)$ is retained.

Step 5-4 Case2-2: $s 2$ has no candidate value, and the candidate for $s 1$ is already determined. Since there is no other competitor, it is determined that $s 2$ is $u k 2$, i.e., $C_{s 2}=(u k 2,1)$.

Step 6: It is determined that $s 1=u k 1$ and $s 2=u k 2$.

(Example 2)

Steps 1 to 4: Generate set $\left\{L_{s i, u k j} \mid i=1 \sim 2, j=1 \sim 2\right\}$. The value of each element is shown below.

\begin{tabular}{|c|c|c|}
\hline Target $s$ & $u k 1$ & $u k 2$ \\
\hline$s 1$ & 3 & 4 \\
\hline$s 2$ & 2 & 1 \\
\hline
\end{tabular}

Step 5-1: Search column $u k l$ for the minimum value. Target $s 2$ has the minimum value. Generate $C_{s 2}=(u k 1,2)$.

Step 5-2 Case2: Move to column $u k 2$. Since $s 2$ has a candidate value, compare $L_{s 2, u k 2}=1$ with $\mathrm{C}_{\mathrm{s} 2}$ (distance value) $=2$. Since $L_{s 2, u k 2}$ is smaller, clear the candidate value for $s 2$. 
Step 5-2: Neither $s 1$ nor $s 2$ has a candidate value. They are competitors. Search column $u k 2$ for the minimum value. Target $s 2$ has the minimum value. Generate $\mathrm{C}_{\mathrm{s} 2}=(u k 2,1)$.

Step 5-4 Case2-1: Go back to $u k l$, and perform the determination of the candidate again. Since $s 2$ has a candidate value, compare $L_{s 2, u k l}=2$ with $C_{s 2}$ (distance value $=1$. Since $C_{s 2}$ (distance value) is smaller, it is determined that $s 2$ is $u k 2$.

Step 5-4 Case2-2: Since the candidate for $s 2$ is already determined, there is no other competitor. Therefore, it is determined that $s 1$ is $u k l$.

Step 6: It is determined that $s 1=u k 2$ and $s 2=u k 1$.

\section{Development of an Experimental System for Evaluation}

We used two smartphones from Panasonic: ELUGA V P-06D. Bluetooth was used for short-range wireless communication. The main specifications of ELUGA V P-06D related to this paper are shown in Table 1. Android Studio was used for the development environment. The application program for the algorithm proposed in Section 2 was implemented in Java. The application program was launched by Android OS. The smartphones synchronized with each other using Bluetooth communication. Location information was obtained via a Wi-Fi AP (access point). Identifiers were displayed using $\mathrm{AR}$ and face detection functions. The experimental system has two user interfaces: the camera screen and the touch screen. GPS information and calculated display positions were also displayed on the camera screen using AR. The configuration of the experimental system software is shown in Fig. 6. GPS coordinates were exchanged via a wireless access point assuming that this method will be used in indoor events, such as offline meetings.

\subsection{P2P Communication Sequence}

Each person inputs his/her identifier, and $\operatorname{logs}$ in. When the terminal search is tapped, a screen for selecting terminals is displayed. When a target terminal is tapped, a connection is set up between this terminal and the target terminal. Each terminal acquires its GPS location information and sends it along with the identifier. Each terminal determines the target's identifier display position from the identifier display coordinates, which has been calculated from the received information, and the face detection coordinates, and displays the identifier using AR. This communication sequence is shown in Fig. 7.

Table 1 Main specifications and design conditions of the smartphone.

\begin{tabular}{|l|l|}
\hline Item & Specification \\
\hline Product name & ELUGA V P-06D (Panasonic) \\
\hline Development environment & Android 4.0 \\
\hline Short-range communication & Android Studio, SDK4 \\
\hline Face detection function & Bluetooth 3.0 (profile: OPP) \\
\hline Wireless LAN & The FaceDetectionListener class was used. \\
\hline GPS acquisition function & IEEE802.11b/g/n \\
\hline Display (resolution) & NETWORK_PROVIDER of the LocationManager class via Wi-Fi was used. \\
\hline Setting of camera display coordinates & $\begin{array}{l}\text { O.6-inch HD(720 } \times 1280 \text { pixels) } \\
\text { x coordinate: The part above the origin is positive. The part below the origin is negative. } \\
\text { y coordinate: The part above the origin is negative. The part below the origin is positive. }\end{array}$ \\
\hline $\begin{array}{l}\text { Constant for conversion from the } \\
\text { relative spatial position to the identifier } \\
\text { display coordinates }\end{array}$ & $\begin{array}{l}\text { K }=20 \\
y_{\text {fix }}=50 \text { pixel }\end{array}$ \\
\hline
\end{tabular}




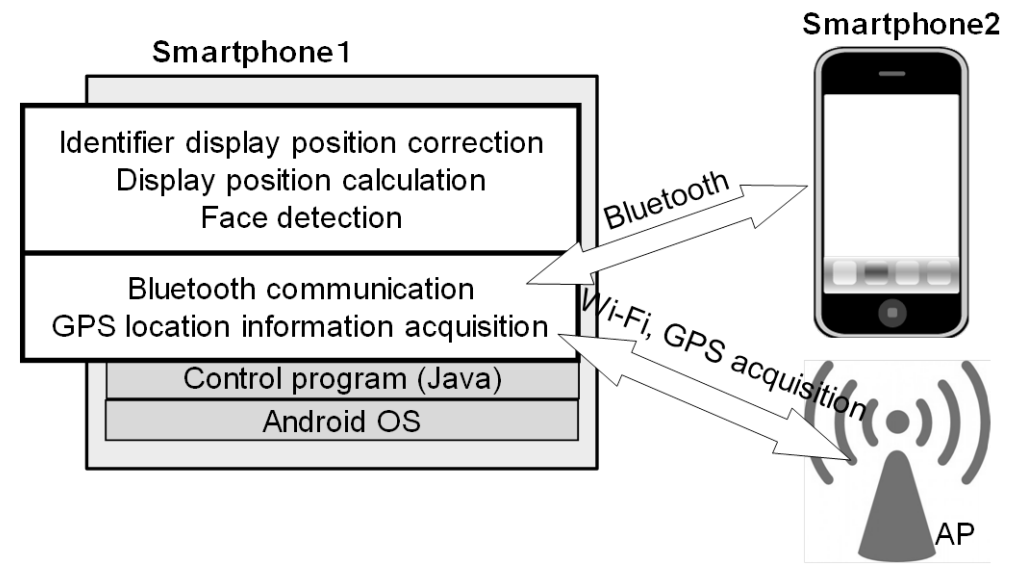

Fig. 6 Configuration of the experimental system software.

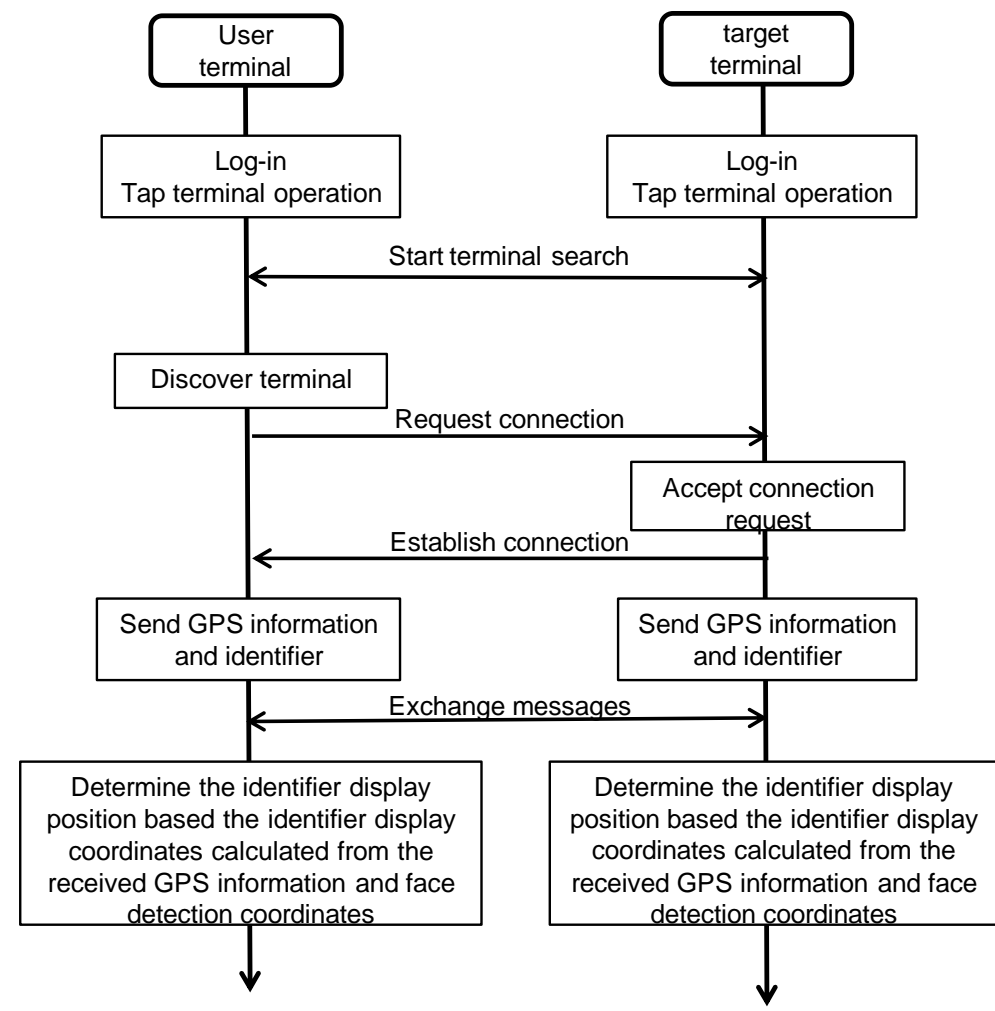

Fig. 7 P2P communication sequence.

\subsection{Operation Example of the Experimental System}

In order to establish Bluetooth communication, the user taps "Terminal search" from the list of icons shown in the upper right corner of Fig. 8. Then, a screen for selecting a terminal appears. The user taps a target terminal to establish the connection to the target. The user taps "Location acquisition" in the upper right corner of Fig. 8 to acquire its location information and

taps the "Send" button to send his/her identifier and location information to the target terminal.

The user taps "Camera" in the upper right corner to launch the camera. At the same time, the terminal calculates the angle to the target from the target's location information, and converts this information into a relative spatial position. An example of usage is shown in Fig. 9. In this case, the distance to the target was $1 \mathrm{~m}$. The target's identifier was "test." This identifier 
was displayed above the face detected using the camera, as shown in Fig. 9a. Fig. 9b shows the relation between $P_{\text {test }}$ and $F_{\text {test }}$ on an x-y plane. Although the position of $P_{\text {test }}$ is outside the camera screen because of GPS errors, the algorithm described in Section 2.4 corrected the display position and the identifier was shown near the head of the target. Even when the target is so far away that his/her face cannot be detected, the use of zooming makes face detection possible. For example, even when the target was $5 \mathrm{~m}$ away as in Fig. 10a, the use of zooming allowed face detection, and the target's identifier was displayed.

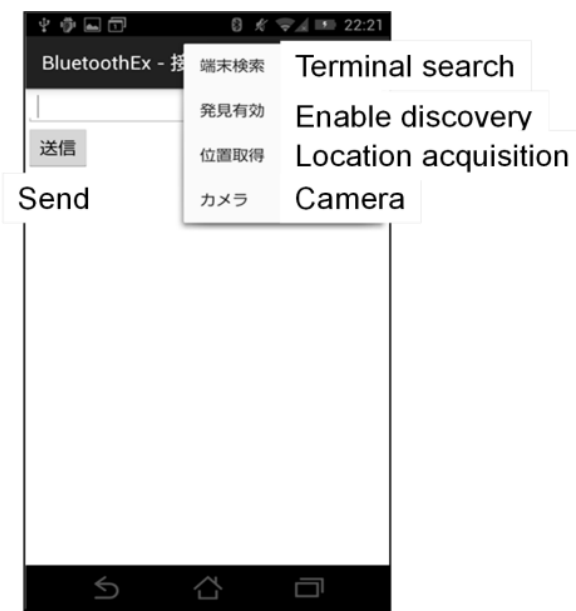

Fig. 8 Initial screen.

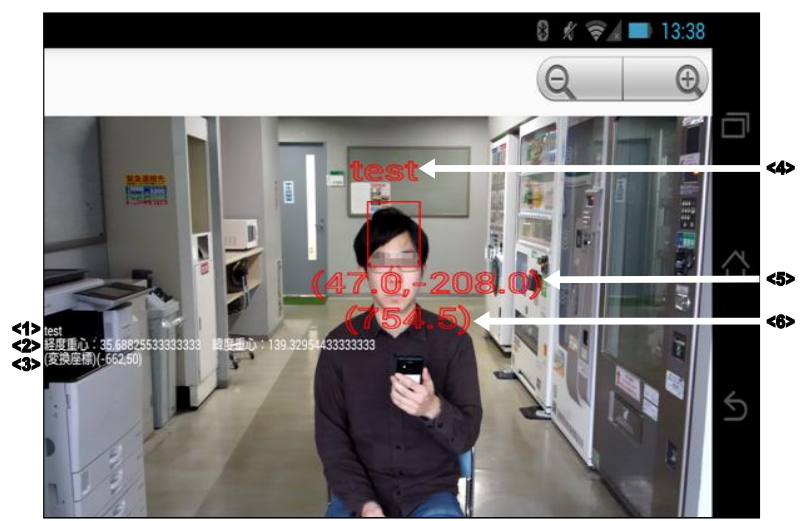

(Note) $<1>$ Identifier of the target whose information has been received. $<2>$ Center of gravity in longitude and that in latitude of the target whose information has been received.

$<3>$ Converted relative spatial position $\mathrm{P}_{\text {test }}<4>$ Identifier of the target displayed. $<5>$ Face detection coordinates $\mathrm{F}_{\text {test. }} \quad\left\langle 6>\right.$ Distance between $\mathrm{F}_{\text {test }}$ and $\mathrm{P}_{\text {test }}$.

(a) Camera screen example

Fig. 9a Identifier is displayed near the head using AR.

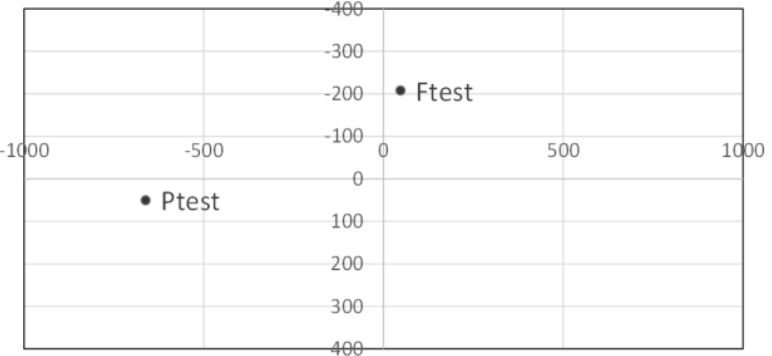

(b) Relation between $\mathrm{P}_{\text {test }}$ and $\mathrm{F}_{\text {test }}$

Fig. 9b Identifier is displayed near the head using AR.

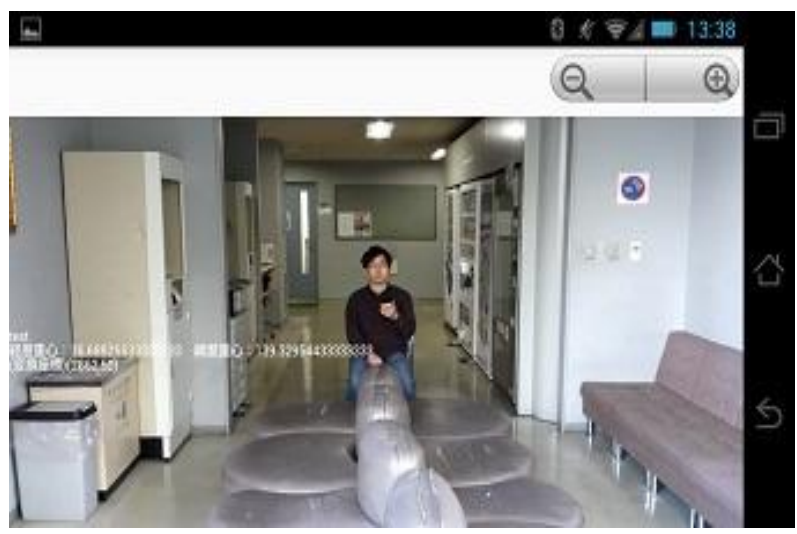

(a) Without zooming
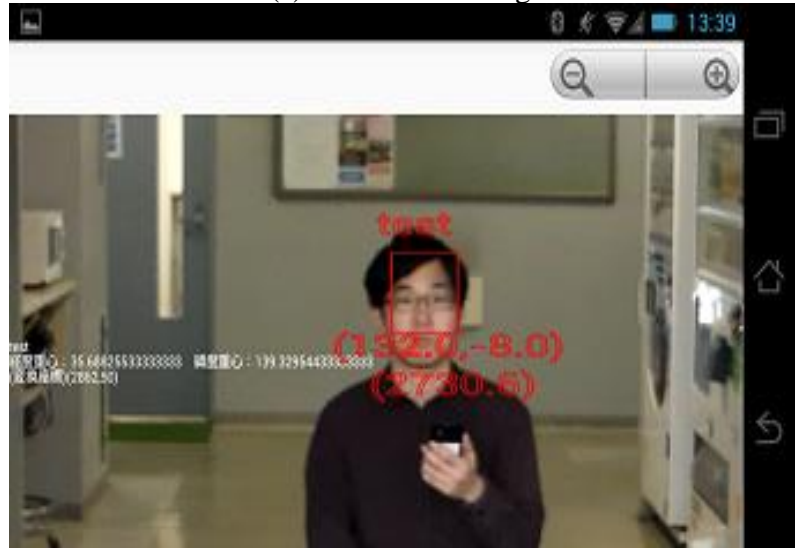

(b) With zooming

Fig. 10 Usage example when the target is $5 \mathbf{m}$ away.

When two faces are detected, each identifier is displayed near the head that is found to be closer based on the coordinates into which the acquired location information has been converted and the face detection coordinates. In the case of Fig. 11, for example, the user has received a message only from Target $A$ but two faces, A and B, have been detected. Target A's identifier "test" is displayed near A, who is closer to the coordinates into which A's location information has been converted. 


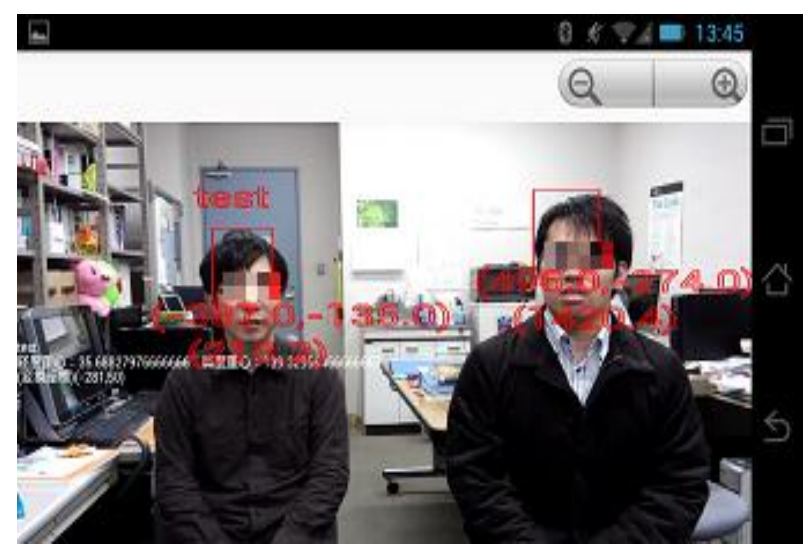

Fig. 11 Usage example when two faces are detected.

\section{Evaluation with Experiments}

Using the experimental system, we evaluated the proposed method for a number of location relations of two targets.

\subsection{Evaluation Conditions}

The experiment conditions are shown in Table 2. Because of the constraints of the experimental system, it was not possible to set up simultaneous connections to multiple target terminals. Therefore, one target terminal was shared by two target persons. After Target A followed the sequence shown in Fig. 7, he tapped the "Return" button. This brought the terminal back to the initial log-in window and disconnected (logged out) the Bluetooth connection. After Target A confirmed the disconnection, he handed the terminal to Target B. Target B followed the sequence shown in Fig. 7.

\subsection{Results of the Experiments Regarding the Relative Locations of Two Targets}

Experiments were conducted for four relative locations of two targets. In Pattern 1, Target A (tarou) and Target B (jirou) were side by side. In Pattern 2,
Target A was in front of the user while Target B was behind the user. In Pattern 3, both targets were in the same screen but Target A was $1 \mathrm{~m}$ behind Target B. In Pattern 4, Target A is in front of the user but Target B was to the right of the user. The results for the four patterns are shown in Table 3. In Patterns 2 and 3, the targets cannot fit into one screen. Therefore, the camera was pointed to each target separately.

In all patterns, there was a big difference between the coordinates into which the target's location information was converted and the face detection coordinates. However, the targets were identified and the identifiers (tarou and jirou) were displayed correctly.

\subsection{Error Examples and Accuracy of Identifier Display}

Table 4 shows cases where GPS location information was not accurate enough or the relative positions of the targets were unfavorable.

In the first example, there was another person in front of the target (“テスト” test). This person was identified as the target and the target's identifier was displayed near him. In the second example, the two targets (tarou and jirou) stood side by side. The identification of them was reversed, and thus a wrong identifier was attached to each target.

We examined the degree of accuracy of the proposed method. Ten experiments were carried out for a case where two targets were side by side. The experiment results are shown in Table 5. The identifiers were displayed correctly 6 times and incorrectly 4 times. The accuracy rate was $60 \%$. The rate was not near $100 \%$ because the accuracy of identifier display coordinates highly depends on the accuracy of GPS location information, which was not accurate enough in some cases.

Table 2 Experiment conditions.

\begin{tabular}{|l|l|}
\hline Item & Conditions and values \\
\hline Experiment site & Indoor \\
\hline Number of terminals & 2 terminals (1 for user. The other was shared by 2 targets in the experiment.) \\
\hline Number of subjects & 1 or 2 targets. 1 non-target. \\
\hline Distance from the user to the targets & 1 to $5 \mathrm{~m}$ \\
\hline
\end{tabular}



GPS Information and Face Detection Using Ad hoc and P2P Networking

Table 3 Results of the experiments regarding the relative locations of two targets.

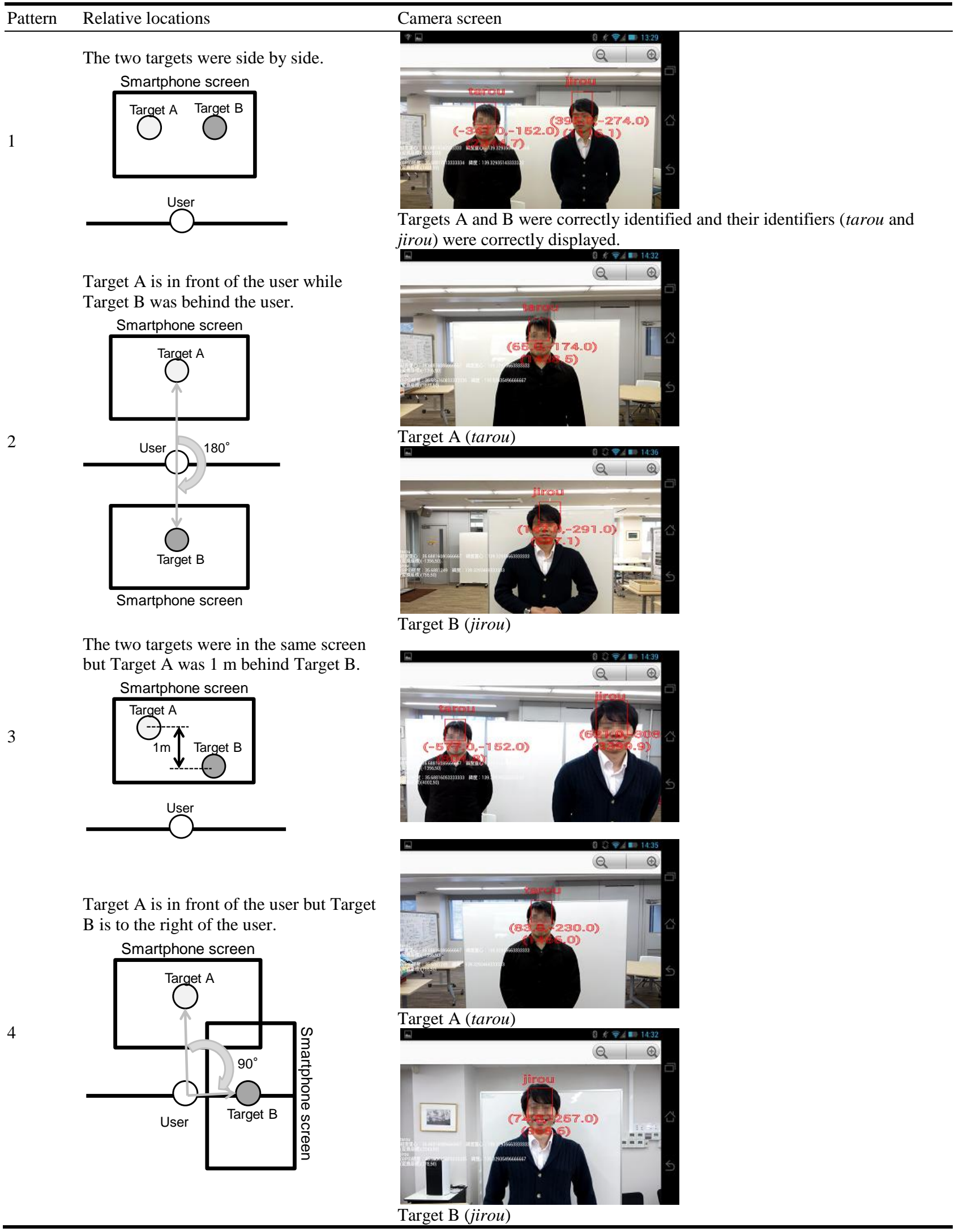


Table 4 Error examples..

Error description
$\begin{aligned} & \text { There was another person in front of the target (“テr } \\ & \text { N"test). The identifier was displayed near this } \\ & \text { person. }\end{aligned}$
$\begin{aligned} & \text { The identification of the two targets (tarou, jirou) was } \\ & \text { reversed. }\end{aligned}$

Table 5 Results of 10 experiments for examining display accuracy.

\begin{tabular}{|c|c|c|c|c|c|c|c|c|c|c|c|c|c|c|c|c|c|}
\hline \multirow{4}{*}{$\begin{array}{c}\text { Number of } \\
\text { experiments }\end{array}$} & \multicolumn{7}{|c|}{ Experiment data } & Assessment & Number of & \multicolumn{7}{|c|}{ Experiment data } & Assessment \\
\hline & \multirow{3}{*}{\multicolumn{3}{|c|}{ FDC }} & \multicolumn{2}{|c|}{ uk1 } & \multicolumn{2}{|c|}{$u k 2$} & \multirow{7}{*}{ Good } & \multirow{7}{*}{ 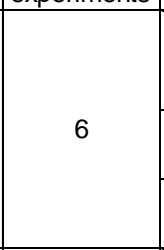 } & \multirow{3}{*}{\multicolumn{3}{|c|}{ FDC }} & \multicolumn{2}{|c|}{$u k 1$} & \multicolumn{2}{|c|}{$u k 2$} & \multirow{7}{*}{ Good } \\
\hline & & & & $x$ & $\mathrm{y}$ & $x$ & $y$ & & & & & & $x$ & $y$ & $x$ & $y$ & \\
\hline & & & & -347 & -152 & 395 & -274 & & & & & & -358 & -19 & 408 & -219 & \\
\hline \multirow[t]{4}{*}{1} & \multirow{2}{*}{ tarou } & $\mathrm{x}$ & -2683 & \multicolumn{2}{|c|}{2345} & \multirow{2}{*}{\multicolumn{2}{|c|}{3095}} & & & \multirow{2}{*}{ tarou } & $\mathrm{x}$ & -2584 & 22 & 27 & 30 & & \\
\hline & & $y$ & 50 & \multirow{3}{*}{\multicolumn{2}{|c|}{1821}} & & & & & & $\mathrm{y}$ & 50 & \multicolumn{2}{|c|}{ tarou } & & & \\
\hline & \multirow{2}{*}{ jirou } & $\mathrm{x}$ & 1463 & & & \multirow{2}{*}{\multicolumn{2}{|c|}{1116}} & & & \multirow{2}{*}{ jirou } & $\mathrm{x}$ & 2832 & 31 & & 24 & & \\
\hline & & $y$ & 50 & & & & & & & & $y$ & 50 & & & jirc & & \\
\hline & & & FDC & uk & & & 2 & & & & & FDC & uk & & $u k$ & & \\
\hline & & & & $x$ & $y$ & $\mathrm{x}$ & $\mathrm{y}$ & & & & & & $x$ & $y$ & $x$ & $y$ & \\
\hline & IDC & & & -353 & -119 & 491 & -202 & & & IDC & & & -325 & -157 & 395 & -374 & \\
\hline 2 & tarou & $\mathrm{x}$ & 1279 & 16 & & & & $\mathrm{Bad}$ & 7 & torou & $\mathrm{x}$ & -2847 & 25 & 30 & 32 & & $\mathrm{Bad}$ \\
\hline & larou & $y$ & 50 & & & & & & & larou & $y$ & 50 & & & $\operatorname{tar}$ & & \\
\hline & & $\mathrm{x}$ & -2362 & 20 & & & & & & & $\mathrm{x}$ & -983 & 69 & & 14 & & \\
\hline & jIrou & $y$ & 50 & jirc & & & & & & Jirou & $y$ & 50 & jirc & & & & \\
\hline & & & FDC & uk & & & 2 & & & & & FDC & uk & & $u k$ & & \\
\hline & & & & $x$ & $\mathrm{y}$ & $x$ & $\mathrm{y}$ & & & & & & $\mathrm{x}$ & $y$ & $\mathrm{x}$ & $\mathrm{y}$ & \\
\hline & IDC & & & -428 & 13 & 423 & -157 & & & IDC & & & -387 & -102 & 390 & -308 & \\
\hline 3 & tarou & $\mathrm{x}$ & -1843 & 14 & & 22 & & Good & 8 & tarout & $\mathrm{x}$ & -2808 & 24 & & 32 & & $\mathrm{Bad}$ \\
\hline & larou & $\mathrm{y}$ & 50 & $\operatorname{tar}$ & & & & & & larou & $y$ & 50 & & & $\operatorname{tar}$ & & \\
\hline & & $\mathrm{x}$ & 886 & 13 & & & & & & & $\mathrm{x}$ & -2737 & 23 & & 31 & & \\
\hline & jIrou & $y$ & 50 & & & & & & & Jirou & $y$ & 50 & jirc & & & & \\
\hline & & & FDC & uk & & & 2 & & & & & FDC & uk & & $u k$ & & \\
\hline & & & & $x$ & $\mathrm{y}$ & $x$ & $\mathrm{y}$ & & & & & & $\mathrm{x}$ & $y$ & $\mathrm{x}$ & $\mathrm{y}$ & \\
\hline & IDC & & & -365 & -86 & 395 & -208 & & & IDC & & & -415 & -208 & 426 & -308 & \\
\hline 4 & tarou & $\mathrm{x}$ & -2428 & 20 & & 28 & & Good & 9 & tarou & $x$ & -3002 & 26 & & 34 & & Good \\
\hline & larou & $\mathrm{y}$ & 50 & $\operatorname{tar}$ & & & & & & larou & $y$ & 50 & $\operatorname{tar}$ & & & & \\
\hline & & $\mathrm{x}$ & -2791 & 24 & & & & & & & $x$ & 2521 & 29 & & 212 & & \\
\hline & jirou & $y$ & 50 & & & & & & & jirou & $y$ & 50 & & & jirc & & \\
\hline & & & FDC & uk & & & 2 & & & & & FDC & uk & & $u k$ & & \\
\hline & & & & $x$ & $y$ & $x$ & $y$ & & & & & & $x$ & $y$ & $x$ & $y$ & \\
\hline & IDC & & & -315 & -263 & 414 & -430 & & & IDC & & & -315 & -174 & 466 & -313 & \\
\hline 5 & tarou & $x$ & -1720 & 14 & & & & Good & 10 & & $x$ & -2305 & 20 & & 27 & & Bad \\
\hline & tarou & $y$ & 50 & $\operatorname{tar}$ & & & & & & tarou & $y$ & 50 & & & $\operatorname{tar}$ & & \\
\hline & & $x$ & 2423 & 27 & & & & & & & $x$ & -1016 & 73 & & 15 & & \\
\hline & jIrou & $y$ & 50 & & & & & & & jirou & $y$ & 50 & jirc & & & & \\
\hline
\end{tabular}




\section{Conclusions and Future Issues}

This paper has proposed a method of displaying the identifier of each target person near his/her head on the camera screens of the smartphones. The smartphones exchange their location information and identifiers of their owners using short-range wireless communication. The paper has proposed to correct identifier display positions using face detection coordinates in order to make up for errors in GPS location measurements. The proposed method was implemented on Android terminals, and experiments were conducted indoors with two target persons. This method determines the identifier display position based the differences in longitude and latitude between the user and the target person and the azimuth direction between the two. This method is sensitive to errors in GPS measurements and the azimuth direction indicated by the electronic compass. It was confirmed that, if these measurements are stable, the correction that uses face detection coordinates yields good results. Identifiers were displayed near the correct target persons at an accuracy rate of $60 \%$.

Looking forward, it will be necessary to improve display accuracy and evaluate the method with more than two target persons. When a third person was between the user and the target person, that person was erroneously recognized as the target person. It will be necessary to improve the method so that the calculation of the identifier display position will take the distance to the target into consideration.

\section{Acknowledgements}

The authors would like to thank our laboratory's members who worked with you on your evaluation experiment.

\section{References}

[1] Investigation Report on the Use Time and the Information Behavior of the Information and Communication Media of 2014, Ministry of Internal
Affairs and Communications of Japan, May 2015. Available online at http://www.soumu.go.jp/menu_news/s-news/01iicp01_02 000028.html.

[2] Contractors Report of Research on Actual Use of Social Media, Ministry of Internal Affairs and Communications of Japan, March 2010. Available online at http://www.soumu.go.jp/johotsusintokei/linkdata/h22_05 _houkoku.pdf.

[3] "NameTag." FacialNetwork.com. Available online at http://www.nametag.ws/.

[4] Avery, B., Thomas, B., and Piekarski, W. 2008. "User Evaluation of See-Through Vision for Mobile Outdoor Augmented Reality." In 7th Int'l Symposium on Mixed and Augmented Reality, 69-72.

[5] Zendjebil, I. M., Ababsa, F., Didier, J.-Y., and Mallem, M. 2008. "Hybrid Localization System for Mobile Outdoor Augmented Reality Applications." First Workshops on Image Processing Theory, Tools and Applications, 1-6, 23-6.

[6] Myung, H., Lee, B., Kim, H., and Kang, S. 2009. “A Study on Effective Visual Communication Method with the 'Pictomation' Contents in Augmented Reality Environment Information Visualization." 13th International Conference Information Visualization, 274-9.

[7] "Sekai Camera." Available online at http://techcrunch.com/2010/07/14/augmented-reality-appsekai-camera-goes-multi-platform-adds-api-and-social-ga ming/.

[8] "Layar." Available online at http://www.fastcompany.com/1441526/layar-augmentedreality-app-hits-200th-data-layer.

[9] Conti, M., Giordano, S., May, M., and Passarella, A. 2010. "From Opportunistic Networks to Opportunistic Computing." IEEE Communications Magazine: 126-39.

[10] Ochiai, H., Shimotada, K., and Esaki, H. 2010. "DTIPN: Delay Tolerant IP Networking for Opportunistic Network Applications." ACM/SIGMOBILE MobiOpp'10, Pisa, Italy.

[11] Pietilainen, A., and Diot, C. 2009. "Experimenting with Opportunistic Networking." ACM International Workshop on Mobility in the Evolving Internet Architecture (MobiArch'09), Krakow, Poland.

[12] Keranen, A., Ott, J., and Karkkainen, T. 2009. "The ONE Simulator for DTN Protocol Evaluation." Second International Conference on Simulation Tools and Techniques (SIMU Tools), Rome, Italy.

[13] Daly, E., and Haahr, M. 2007. "Social Network Analysis for Routing in Disconnected Delay-Tolerant MANETs." ACM International Symposium on Mobile Ad Hoc Networking and Computing (MobiHoc'07), Montreal, Quebec, Canada. 
[14] Burke, J., Estrin, D., Hansen, M., Parker, A., Ramanathan, N., Reddy, S., and Srivastava, M. B. 2006. "Participatory Sensing." Workshop on World-Sensor-Web (WSW'06): Mobile Device Centric Sensor Networks and Applications (WSW'06 at SenSys '06), Boulder, Colorado, USA.
[15] Kawabata, H., and Takami, K. 2012. "A Method of Simultaneously Displaying a Passing Person and His/Her Message on the Same Screen Space.” IEEE International Workshop on the Impact of Human Mobility in Pervasive Systems and Applications 2012 (PerMoby 2012), Lugano, Switzerland. 